\title{
Erratum to: Distribution of surfactant ions near the surface of non-aqueous solution gained by angular resolved $\mathrm{X}$-ray photoelectron spectroscopy
}

\author{
Chuangye Wang $\cdot$ Harald Morgner
}

Published online: 20 November 2010

(C) Springer-Verlag 2010

\section{Erratum to: Microchim Acta}

DOI 10.1007/s00604-010-0446-6

Unfortunately, a misspelling occurred in this paper, both in abstract and in the main text. The word, "generic", should be corrected to be "genetic". The correct Abstract is given below:

Abstract Angular resolved X-ray photoelectron spectroscopy (ARXPS) has been applied to obtain the distribution of chemical elements near the surface of non-aqueous solutions containing surfactants. However, such profiles can only yield a quantitative relation between those constituents near the surface regime of sample. With the knowledge of the molar volumes of surfactant and solvent, we have obtained the molar concentration-depth profiles via the molar fraction-depth profiles that were reconstructed by ARXPS with the help of a genetic algorithm. The concentration profiles show detailed distributions of the surfactant ions near the surface, which provide a direct insight into the surface picture of the surfactant solution.

The online version of the original article can be found at http://dx.doi. org/10.1007/s00604-010-0446-6.

C. Wang $(\bowtie) \cdot$ H. Morgner

Wilhelm-Ostwald Institute for Physical and Theoretical

Chemistry, Department of Chemistry and Mineralogy,

University of Leipzig,

Linnestrasse 2,

04103, Leipzig, Germany

e-mail: chwang@rz.uni-leipzig.de 